\title{
ÁCIDOS GRASOS NO ESTERIFICADOS AL PARTOY SU RELACIÓN CON PRODUCCIÓN LECHERA EN VACAS HOLSTEIN
}

\author{
NON ESTERIFIED FATTY ACIDS AT CALVING AND ITS RELATIONSHIP WITH MILK \\ YIELD IN HOLSTEIN COWS
}

\author{
Marín, M.P. ${ }^{1 *}$, Ríos, C. ${ }^{1 A}$, Contreras, $H .{ }^{2}$, Robles, J. ${ }^{1}$ y Meléndez, P. ${ }^{1 B, 3}$ \\ ${ }^{1}$ Universidad Santo Tomás. Facultad de Medicina Veterinaria. Av. Limonares 190. Viña del Mar. Chile. \\ *mmarin@santotomas.cl; ${ }^{A}$ crios@santotomas.cl; ${ }^{B}$ pedro.melendez@santotomas.cl \\ 2Bioleche. Av. Las Industrias 510. Los Angeles. Chile. hcontreras@bioleche.cl \\ ${ }^{3}$ College of Veterinary Medicine. University of Florida. Gainsville, FL 32610. EEUU.
}

\section{Palabras clave adicionales}

Lactación. Reservas corporales.

\section{RESUMEN}

El objetivo de este estudio fue determinar la relación entre ácidos grasos no esterificados (AGNE) al parto, reservas corporales (CC) y producción de leche en vacas Holstein en Chile central. Este estudio se realizó en dos rebaños lecheros comerciales con una producción promedio de leche de $8000 \mathrm{~kg}$ por lactación. Las vacas fueron alimentadas con ración completa basada en heno de alfalfa (heno y cortada en verde), ensilaje de maíz y concentrado comercial. Se obtuvieron muestras de sangre de 350 vacas al momento del parto para la determinación de AGNE. Se determinó $\mathrm{CC}$ al parto y a los 60 días posparto y producción de leche acumulada a los 30,60 y 100 días.

Los datos fueron analizados utilizando el procedimiento de modelos mixtos de SAS (2003). Los valores promedio de AGNE al parto y la CC fueron de $0,90 \pm 0,47 \mathrm{meq} / \mathrm{l}$ y $3,6 \pm 0,3$, respectivamente.

Considerando la $\mathrm{CC}$ al parto y el número de partos como variables independientes en un modelo multivariado, la relación entre los AGNE al parto y la producción de leche a los 100 días de lactación fue de tipo cuadrática, es decir, para valores tanto bajos como altos de AGNE, la producción acumulada fue menor en comparación a niveles intermedios $\left(R^{2}=0,27 ; p<0,001\right)$. Se observó una correlación positiva entre CC y AGNE al parto $(r=0,26 ; p<0,05)$. Se determinó una tendencia a que en la medida que los valores de AGNE son mayores, el cambio en la CC es mayor. Se conclu-

\section{AdDitional KeYWORDS}

Milk yield. Body condition.

ye que los AGNE al parto se asocian en forma cuadrática con la producción de leche acumulada a los 100 días.

\section{SUMMARY}

The purpose of this study was to determine the relationship between non-esterified fatty acids (NEFA) at calving, body condition score (BCS), and milk yield in Holstein cows from central Chile. The study was conducted on two commercial dairy herds with an average milk yield of $8000 \mathrm{~kg}$ per lactation. Cows were fed a total mixed ration based on alfalfa hay, corn silage and concentrate. Blood samples were taken from 350 cows at calving and NEFA were determined. BCS at calving and at 60 days post partum, accumulated milk yield at 30,60 and 100 days of lactation were determined.

Data were analyzed using the mixed procedure of SAS (2003). Mean values for NEFA at calving, and BCS were $0.90 \pm 0.47 \mathrm{meq} / \mathrm{l}$, and $3.6 \pm 0.3$, respectively.

In a multivariable level model, considering BCS at calving and parity as independent variables, the relationship between NEFA at calving and milk yield at 100 days of lactation was quadratic, with lower milk yield at high and low values of NEFA and higher milk yield at intermediate levels of $\operatorname{NEFA}\left(R^{2}=\right.$ $0.27 ; p<0.001)$. A positive correlation was observed between BCS and NEFA at calving $(r=$ $0.26 ; p<0.05$ ). Concentration of NEFA increased 
when the change in BCS between calving and 60 days post calving was higher. It is concluded that NEFA at calving, was associated in a quadratic form with milk yield at 100 days of lactation.

\section{INTRODUCCIÓN}

El balance energético es el resultado entre las necesidades del animal y los aportes alimentarios. Durante las últimas 2 a 4 semanas de gestación se produce un aumento sustancial de las necesidades energéticas acompañado de una disminución en la ingesta de materia seca, fundamentalmente en la última semana preparto (Grummer, 1995; Goff y Horst, 1997). Estas dos circunstancias son responsables del balance energético negativo que se inicia unas semanas antes del parto. Lo anterior implica una movilización de grasa corporal y el consiguiente aumento de los ácidos grasos no esterificados (AGNE) en sangre, especialmente desde 2 a 3 días antes del parto, alcanzando su máximo al momento del parto (Meléndez et al., 2002). Los AGNE son utilizados por el hígado como fuente de energía (Drackley et al., 2006), reesterificados a triglicéridos hepáticos y exportados nuevamente a circulación en la forma de lipoproteínas de muy baja densidad(VLDL). No obstante, si los niveles de glucosa son reducidos, los AGNE son convertidos a cuerpos cetónicos, los que al incrementarse sobre los niveles normales, causan el cuadro conocido como cetosis (Grummer, 1993). Por otro lado, la excesiva entrada de AGNE al hígado puede provocar un aumento de triglicéridos con el consecuente desarrollo de hígado graso (Meléndez y Risco, 2005). Se ha demostrado que vacas con elevados niveles de AGNE en sangre durante el preparto y el parto experimentan una mayor incidencia de enfermedades del periparto (Dyk et al., 1995). Por otro lado, Goff y Horst (1997), sugieren que una pérdida excesiva de condición corporal se asocia con un aumento de los niveles de AGNE que, a su vez, se relaciona con una reducción en la fertilidad y producción de leche. Sin embargo, son escasos los estudios que han asociado niveles de AGNE al parto con producción de leche. La hipótesis de este estudio es que la producción de leche se ve afectada según los valores de AGNE al momento del parto. Por lo tanto, el objetivo de este trabajo fue relacionar las concentraciones séricas de AGNE al parto con la producción de leche durante los primeros 100 días de lactancia en ganado Holstein de la zona centrosur de Chile.

\section{MATERIAL Y MÉTODOS}

El estudio se llevó a cabo en 2 planteles lecheros de raza Holstein de la zona centro y centro sur de Chile, con un nivel de producción de aproximadamente $9000 \mathrm{~kg}$ de leche por lactación y mantenidos en confinamiento todo el año. La alimentación consistió en alfalfa (heno y cortada en verde), ensilaje de maíz y concentrado comercial, suministrada como ración completa. Cada plantel contaba con un sistema de registros individuales productivos y reproductivos.

Con el propósito de encontrar una diferencia de 1 litro en producción de leche con una desviación estándar de 3,5 litros entre vacas con elevados niveles de AGNE y vacas con valores normales de AGNE, con un nivel de confianza del $95 \%$ y un poder del test estadístico del $80 \%$, se calculó un tamaño muestral de 310 animales (Winepiscope $2.0,2000)$. Se seleccionaron 350 vacas al azar al momento del parto, determinando de forma inmediata su CC, utilizando una escala de 1 a 5 según la metodología propuesta por Ferguson et al. (1994). Se determinó, de nuevo, la CC a los 60 días, para estimar el cambio de la CC durante este período.

Se registró la producción de leche acumulada a los 30, 60 y 100 días de lactancia. Se obtuvo una muestra de sangre al parto desde el plexo sanguíneo de la base de la cola utilizando tubos al vacío (Vacutainer ${ }^{\circledR}$ ), para determinar las concentraciones de AGNE (meq/l). Para ello las muestras fueron 
ÁCIDOS GRASOS NO ESTERIFICADOS Y PRODUCCIÓN LECHERA EN HOLSTEIN

Tabla I. Producción de leche acumulada $(\mathrm{kg})$ a los 30, 60 y 100 días posparto. (Accumulated milk yield (kg) up to 30,60 and 100 days postpartum).

\begin{tabular}{ccccccr}
\hline Días posparto & $\mathrm{n}$ & Promedio & Mediana & Desviación estándar & Mínimo & Máximo \\
\hline 30 & 350 & 1019,70 & 1020,00 & 266,60 & 210 & 1780,00 \\
60 & 350 & 2181,84 & 2190,00 & 557,47 & 324 & 3460,00 \\
100 & 344 & 3725,41 & 3750,00 & 892,84 & 429 & 5960,00 \\
\hline
\end{tabular}

centrifugadas (3000 rpm durante $10 \mathrm{minu}-$ tos) para la obtención del suero, que se almacenó a $-20^{\circ} \mathrm{C}$ hasta su posterior análisis mediante un método enzimático colorimé-

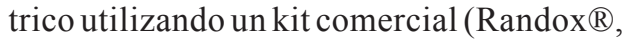
UK) y cuantificadas a través de un método de espectrofotometría (Microlab 200, Merck ${ }^{\circledR}$ ).

Las distintas variables fueron analizadas a través de estadística descriptiva. Se calcularon media, desviación estándar, rango y otros parámetros de variación.

Para el análisis de los AGNE al parto, se estableció un modelo mix to lineal (Littell et al., 2002) considerando como variables independientes la $\mathrm{CC}$ al parto y el número de lactancia.

Para el análisis de la producción acumulada a los 100 días se desarrolló un modelo mixto lineal, considerando como variable independiente principal a los AGNE al parto, corrigiendo por $\mathrm{CC}$ al parto y el número de lactancia.

Los análisis fueron llevados a cabo utilizando el programa estadístico SAS (2003) con un límite de confianza de $95 \%$, según su respectivo procedimiento.

\section{RESULTADOSYDISCUSIÓN}

La estadística descriptiva para los valores promedio de la producción de leche acumulada $(\mathrm{kg})$ a los 30, 60 y 100 días de lactancia, AGNE al parto (meq/1), y la CC obtenidas al parto y a los 60 días posparto, así como el cambio en la CC en el período, se presentan en las tablas I y II, respectivamente.

El promedio del número ordinal de partos fue de 2,34 $\pm 1,47$, indicando que la mayoría son vacas multíparas $(66 \%)$. Los niveles de producción se ajustan a vacas de alta producción, con una producción láctea máxima acumulada a los 100 días posparto de $5960 \mathrm{~kg}$. Es decir, la muestra en estudio obtuvo una producción diaria de leche en promedio hasta los 30 días posparto de aproximadamente $33 \mathrm{~kg}$, alcanzando a los 100 días posparto un promedio diario de 37 $\mathrm{kg}$. Esto concuerda con el estudio realizado por Davis et al. (2003), quienes señalan que vacas de alta producción en sistemas intensivos en Chile logran una producción de leche promedio, en un lapso de 305 días de lactancia de $9323 \mathrm{~kg}$.

Tabla II. Ácidos grasos no esterificados al parto y condición corporal al parto (CCp) y a los 60 días posparto (CC60). (Non esterified fatty acids at calving and body condition score at calving and at 60 days postpartum).

\begin{tabular}{lcccccc}
\hline \multicolumn{1}{c}{ Variable } & $\mathrm{n}$ & Promedio & Mediana & Desviación estándar & Mínimo & Máximo \\
\hline AGNE (meq/l) & 333 & 0,909 & 0,860 & 0,474 & 0,150 & 2,450 \\
CCp & 353 & 3,62 & 3,75 & 0,34 & 2,25 & 4,25 \\
CC60 & 347 & 3,13 & 3,25 & 0,41 & 2,0 & 4,0 \\
Cambio entre CCp y CC60 & 347 & $-0,53$ & 0,5 & 0,48 & - & - \\
\hline
\end{tabular}




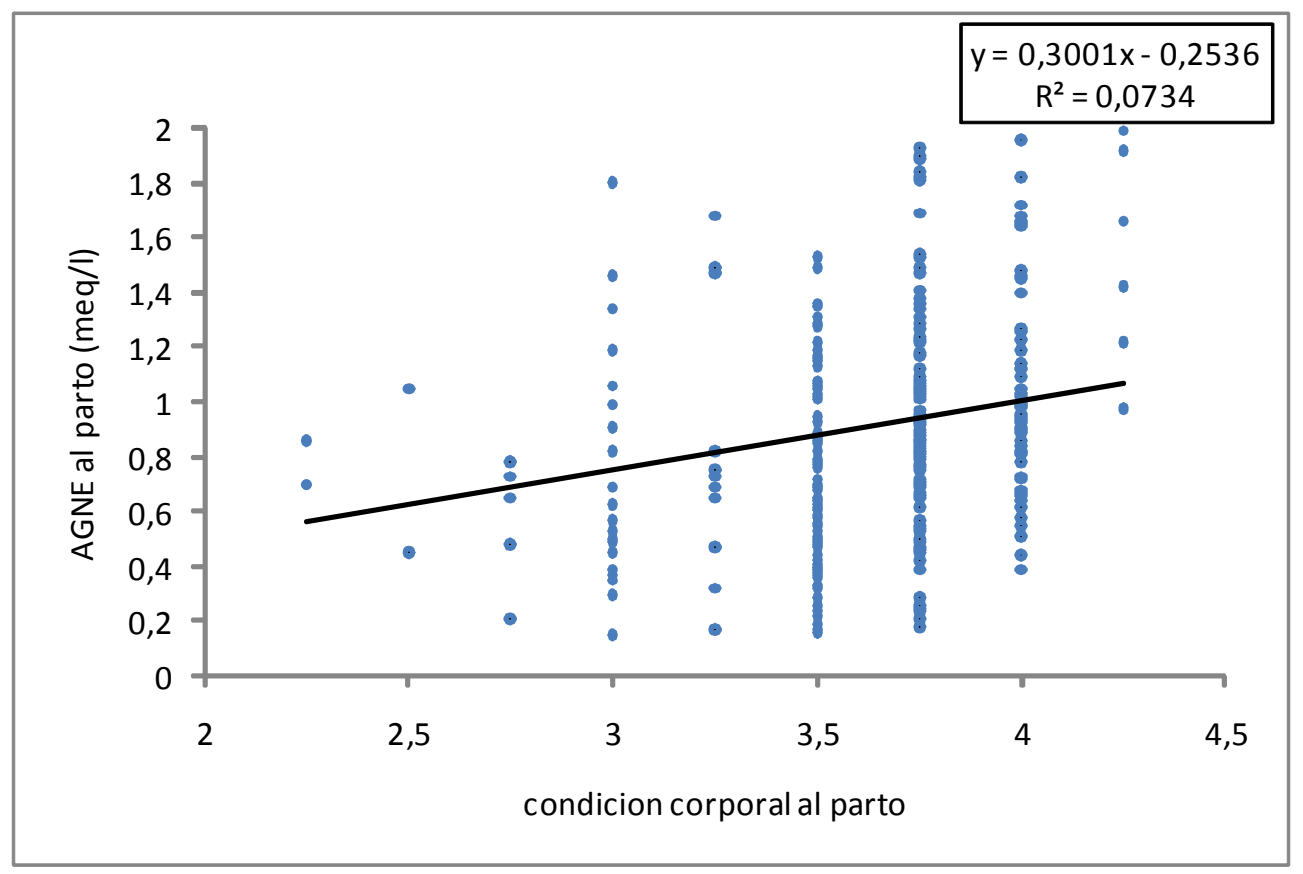

Figura 1. Relación entre la condición corporal al parto y los niveles de AGNE al parto. (Relationship between body condition score and non esterified fatty acids at calving).

Con respecto a los AGNE al parto, el valor promedio en este estudio fue de 0,9 $\mathrm{meq} / \mathrm{l}$ con un rango entre 0,15 y $2,45 \mathrm{meq} / 1$, lo que indica una amplia variabilidad en los resultados. Este valor promedio corresponde al límite inferior descrito por Meléndez et al. (2002), quienes establecen como concentración máxima de AGNE al parto 1,2 $\mathrm{meq} / \mathrm{l}$ y un rango de $0,9-1,2 \mathrm{meq} / \mathrm{l}$. Drackley et al. (2001), describen valores de 0,8 a 1,2 $\mathrm{meq} / \mathrm{l}$, en el día del parto, con un descenso a $0,7 \mathrm{meq} / \mathrm{l}$ a los 7 días posparto. Cheng et al. (2007), señalan concentraciones de NEFA al día uno posparto, en animales hipoglicémicos, de 1,44 meq/ly de 1,05 meq/l en vacas normales.

La CC al momento del parto y a los 60 días y el cambio observado durante este período sugieren un manejo adecuado. Según Buckley et al. (2003), un plantel con un manejo ideal de la condición corporal de animales de alta producción deberían tener una $\mathrm{CC}$ al parto en promedio de $3,6 \pm 0,37$ puntos y un cambio de la condición corporal entre el parto y 60 días posparto en promedio $0,5 \pm 0,35$ puntos y 1 punto como pérdida máxima. Esto concuerda con Domeq et al. (1997), quienes proponen que una CC entre 3,0 - 3,5 (escala 1 a 5) es la más indicada para el parto y la disminución en el posparto no debería ser superior a 1 unidad e idealmente 0,5 , como pérdida máxima en los primeros 70 días de lactancia, para iniciar la recuperación de las reservas energéticas a los 80-90 días posparto.

En la figura 1 se indica la relación existente entre la CC y los niveles de AGNE, ambos al parto. A medida que aumentó la $\mathrm{CC}$, los niveles de AGNE aumentaron ( $p<0,01)$; es decir, animales que tuvieron una $C C 4,0$ y más, presentaron los niveles mayores de AGNE analizados y en contraste, los anima- 


\section{ÁCIDOS GRASOS NO ESTERIFICADOS Y PRODUCCIÓN LECHERA EN HOLSTEIN}

les de 2,5 de CC al parto, presentaron los niveles más bajos del grupo de animales en estudio. Sin embargo la correlación, a pesar de ser positiva, fue baja, puesto que sólo un $7 \%$ del aumento de los niveles de AGNE al parto fueron explicados por la $\mathrm{CC}$ al parto. Estos resultados concuerdan con los obtenidos por Pedron et al. (1993), quienes indicaron que vacas con una $\mathrm{CC}$ al parto de 4,0 poseían las concentraciones más altas de AGNE, en cambio en vacas con una $\mathrm{CC}$ al parto menor a 3,0, las concentraciones de AGNE fueron inferiores. En general los animales de dicho estudio llegaron al parto con una CC 3,5 hasta 4,0 y los niveles de AGNE permanecieron elevados durante la lactancia.

La relación entre los AGNE y el cambio en la CC entre el parto y los 60 días posparto se presenta en la figura 2. En ésta se puede observar que al aumentar los niveles de AGNE al parto, el cambio de la CC entre el parto y los 60 días posparto tiende a ser mayor, puesto que a mayor pérdida de CC existe una mayor movilización grasa. Esta situación es demostrada en varios estudios, como los realizados por Veenhuizen $e t$ al. (1991) quienes sugieren que la concentración de AGNE en suero es dependiente del nivel de lipólisis en el tejido adiposo y la captación de éstos en hígado y otros tejidos. A su vez el nivel de lipólisis está determinado, entre muchos factores, por el cambio de la CC del animal. Sin embargo, esta asociación es leve, ya que sólo un 1,7\% de la variación en el cambio de la CC fue explicada por los niveles de AGNE al parto.

Con respecto a los AGNE y su relación con producción de leche acumulada a los 100 días (figura 3), se puede observar que ésta fue de tipo cuadrática, lo que implica que con valores tanto bajos como altos de AGNE, la producción acumulada posparto fue menor en comparación a niveles intermedios de AGNE. En este modelo multivariable, considerando $\mathrm{CC}$ al parto, número de

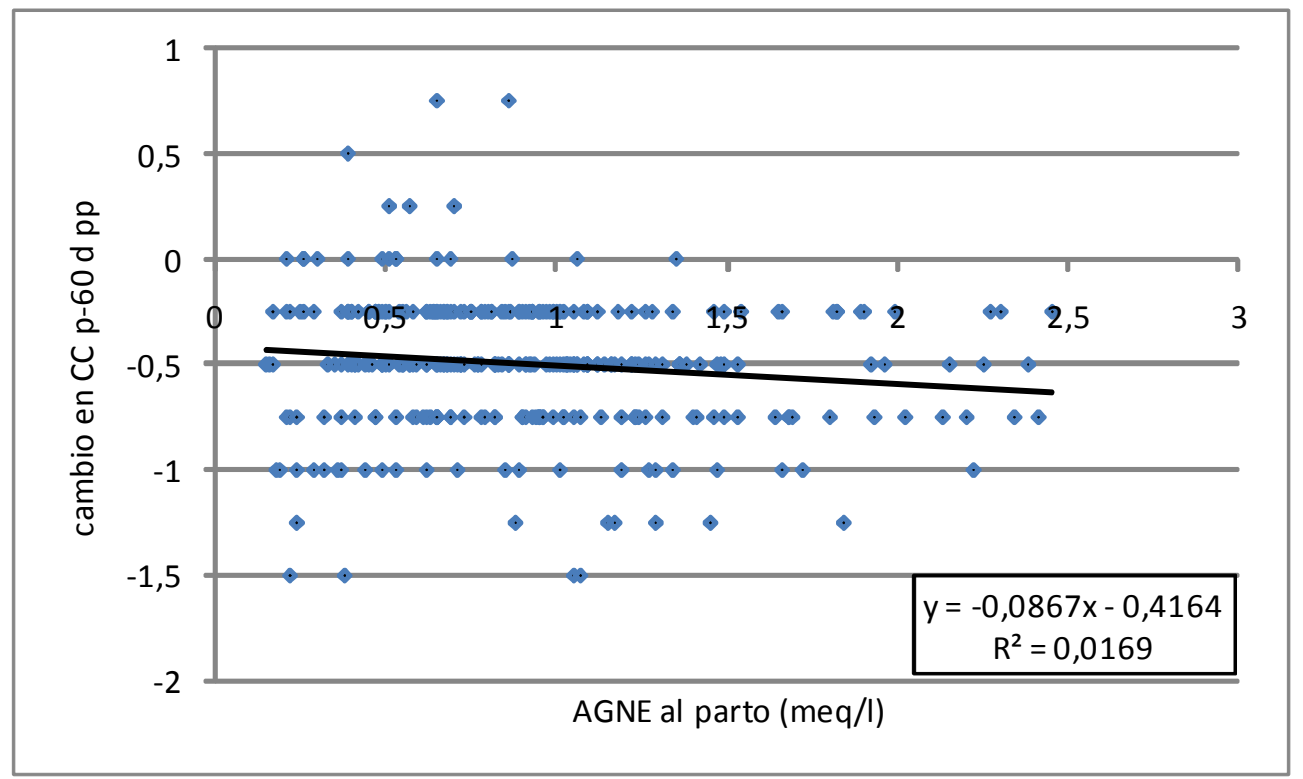

Figura 2. Relación entre los niveles de AGNE al parto y el cambio de la condición corporal entre el parto y 60 días posparto. (Relationship between non esterified fatty acids at calving and the change in body condition score between calving and 60 days postpartum). 


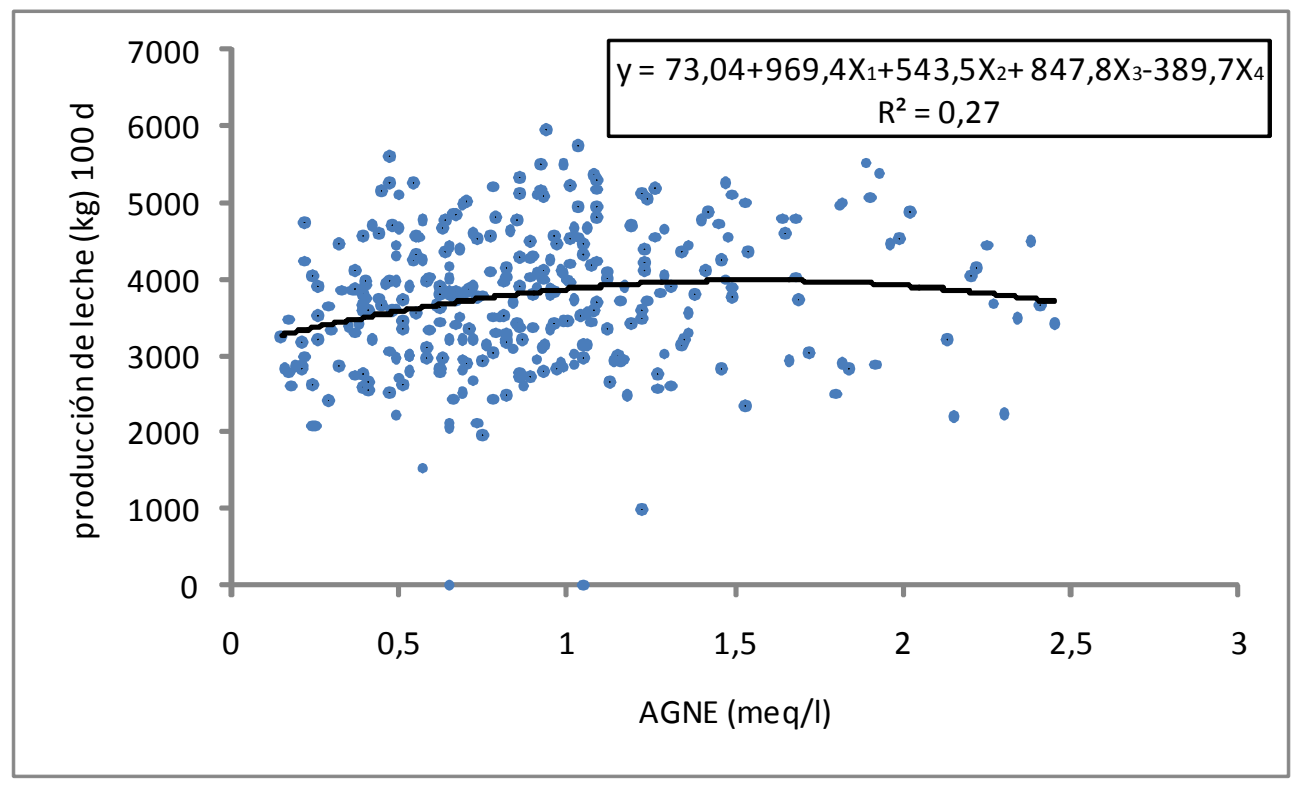

Figura 3. Relación entre los niveles de AGNE al parto con el rendimiento productivo acumulado a los 100 días de lactancia. (Relationship between non esterified fatty acids at calving and accumulated milk yield up to 100 days postpartum).

lactancia y las concentraciones de AGNE al parto, se observó que el $27 \%$ de la variabilidad en la producción de leche acumulada a los 100 días de lactancia fue explicada por este modelo $\left(\mathrm{R}^{2}=0,27\right)$ :

$Y=730,4+969,4 X_{1}+543,5 X_{2}+847,8 X_{3}-389,7 X_{4}$

donde:

$\mathrm{X}_{1}$ : AGNE al parto,

$\mathrm{X}_{2}$ : condición corporal al parto,

$\mathrm{X}_{3}$ : lactancia,

$\mathrm{X}_{4}$ : AGNE al parto $\times \mathrm{AGNE}$ al parto.

Domecq et al. (1997) y Pedron et al. (1993) observaron que una pérdida excesiva de condición corporal ha sido asociada con un aumento de los niveles de AGNE y a su vez esta alza se relaciona con una fertilidad y producción de leche reducida. Sin embargo, en el gráfico no sólo se demuestra que altos niveles de AGNE al parto podrían afectar negativamente la producción, sino que ello también ocurre con niveles bajos de
AGNE al parto, relación quizás poco estudiada en las diferentes investigaciones referidas al tema. Cheng et al. (2007) y Guo et al. (2007) no encontraron diferencias en producción de leche en lactancia temprana, en grupos con diferentes valores de AGNE. En un estudio en que se midió la producción de leche acumulada a los 100 días (Moallem et al., 2007), se encontró un incremento tanto en los niveles de AGNE al parto como en los niveles de producción de leche al adicionar un suplemento rico en ácidos grasos en la ración de los animales. En un estudio realizado por Waltner et al. (1993), donde se analizó la asociación entre la CC al parto y la producción de leche, también se observó una relación cuadrática, los animales tanto con baja CC $(2,75$ o menos $)$ como altas CC al parto (4,0 o más) experimentaron una producción de leche acumulada a los 90 días más baja que animales que parieron con una CC óptima $(3,0$ a 3,75). Si consideramos que en el presente estudio las vacas con 


\section{ÁCIDOS GRASOS NO ESTERIFICADOS Y PRODUCCIÓN LECHERA EN HOLSTEIN}

baja $\mathrm{CC}$ al parto tuvieron un bajo nivel de AGNE y las vacas con una elevada $\mathrm{CC}$ al parto tuvieron altas concentraciones de AGNE, se sugiere que los resultados obtenidos por Waltner et al. (1993) avalan indirectamente los resultados del presente trabajo. No obstante, al considerar la CC al parto dentro del modelo estadístico, se establece que los AGNE al parto, independiente de la $\mathrm{CC}$ al parto y el número de lactancia, tienen una asociación (cuadrática) con la producción de leche acumulada a los 100 días.

\section{CONCLUSIONES}

La concentración de AGNE al parto se

\section{BIBLIOGRAFÍA}

Buckley, F., O'Sullivan, K., Mee, J.F., Evans, R.D. and Dillon, P. 2003. Relationships among milk yield, body condition, cow weight and reproduction in spring-calved HolsteinFriesians. J. Dairy Sci., 86: 2308-2319.

Cheng, X., Zhe,W., Shuling, N., Chuang, X., Cai, Z. and Hongyou, Z. 2007. Effect of hypoglycemia on performances, metabolites and hormones in periparturient dairy cows. Agric. Sci. China, 6: 505-512.

Davis, P., Magofke, J.C., García, X., Gana, E. y Gonzáles, H. 2003. Comparación de dos sistemas de manejo en vacas Holstein Americano de alta producción, X región, Chile. XXVIII Congreso Anual de la Sociedad Chilena de Producción Animal. Talca. Chile.

Domecq, J.J., Skidmore, A.L., Lloyd, J.W. and Kaneene, J.B. 1997. Relationships between body condition scores and milk yield in a large dairy herd of high yielding Holstein cows. J. Dairy Sci., 80: 101-112.

Drackley, J.K., Overton, V. and Douglas, J.N. 2001. Adaptations of glucose and long chain fatty acid metabolism in liver of dairy cows during the periparturiente period. J. Dairy Sci., 84 (Suppl.): E100-E112.

Drackley, J.K., Donkin, S.S and Reynolds, C.K 2006. Major advances in fundamental dairy cattle nutrition. J. Dairy Sci., 89: 1324-1336.

Dyk, P.B., Emery, R.S., Liesman, J.L., Bucholtz, relacionó cuadráticamente con la producción de leche acumulada a los 100 días de lactancia. Vacas con niveles tanto bajos como altos de AGNE al parto, tuvieron una producción acumulada a los 100 días de lactancia, más baja que vacas con niveles intermedios de AGNE.

\section{AGRADECIMIENTOS}

Sociedad Agrícola Alma y al Sr. Roberto Ruff, por facilitar los animales de su plantel lechero.

Fuente de financiación, Proyecto de investigación interno. Universidad Santo Tomás Inv27/06.

H.F. and VandeHaar, M.J. 1995. Prepartum nonesterified fatty acids in plasma are higher in cows developing periparturient health problems. J. Dairy Sci., 78 (Suppl. 1): 264.

Ferguson, J.D., Galligan, D. and Thomsen, N. 1994. Principal descriptors of body condition score in Holstein cows. J. Dairy Sci., 77: 2695-2703.

Goff, J.P. and Horst, R.L.1997. Physiological changes at parturition and their relationship to metabolic disorders. J. Dairy Sci., 80: 12601268.

Guo, J., Peters, R.R. and Köhn, R. A. 2007. Effects of a transition diet on production, performance and metabolism in periparturient dairy cows . J. Dairy Sci., 90: 5247-5258.

Grummer, R.R. 1993. Etiology of lipid-related metabolic disorders in periparturient dairy cows. J. Dairy Sci., 76: 3882-3896.

Grummer, R.R. 1995. Impact of changes in organic nutrient metabolism on feeding the transition dairy cow. J. Anim. Sci., 73: 2820-2833.

Littell, R.C., Stroup, W.W. and Freund, R.J. 2002. SAS for linear models. $4^{\text {th }}$ ed. SAS Institue Inc. Cary, NC. USA.

Meléndez, P., Donovan, A., Risco, C.A., Hall, B.A., Littell, R. and Goff, J. 2002. Metabolic responses of transition cows fed anionic salts and supplemented at calving with calcium and energy. J. Dairy Sci., 85: 1085-1092.

Meléndez, P. and Risco, C.A. 2005. Management 


\section{MARÍN, RÍOS, CONTRERAS, ROBLES Y MELÉNDEZ}

of transtition cow to optimize reproductive efficiency in dairy herds. Vet. Clin. North Am. Food Anim. Prac., 21: 485-501.

Moallem, U., Katz, M., Arielli, A. and Lehrer, H. 2007. Effects of peripartum propylene glycol or fats differing in fatty acid profiles on feed intake, production and plasma metabolites in dairy cows. J. Dairy Sci., 90: 3846-3856.

Pedron, O., Cheli, F., Senatore, E., Baroli, D. and Rizzi, R. 1993. Effect of body condition score at calving on performance, some blood parameters, and milk fatty acid composition in dairy cows. $J$. Dairy Sci., 76: 2528-2535.

SAS. 2003. SAS/STAT Software: Change and enhancements through release 9.0 for Windows. SAS Inst. Inc. Cary, NC.
Veenhuizen, J.J., Drackley, J.K., Richard, M.J., Sanderson, T.D., Miller, L.D. and Young, J.W. 1991. Metabolic changes in blood and liver during develoment and early treatment of experimental fatty liver and ketosis in cows. J. Dairy Sci., 74: 4238-4253.

Waltner, S.S., McNamara, J.P. and Hillers, J.K. 1993. Relationships of body condition score to production variables in high producing Holstein dairy cattle. J. Dairy Sci., 76: 3410-3419.

WinEpiscope 2.0. 2000. Software for quantitative veterinary epidemiology. Facultad de Veterinaria. Zaragoza. Spain. Agricultural University Wageningen. Netherlands. University of Utrecht. Netherlands. University of Edinburgh. United Kingdom. Borland Delphi@ 1.0. 